\title{
Development of a Novel Three-dimensional Deformable Mirror with Removable Influence Functions for High Precision Wavefront Correction in Adaptive Optics System
}

\author{
Lei Huang ${ }^{1 * 2^{*}}$, Chenlu Zhou ${ }^{1 *}$, Mali Gong ${ }^{1}$, Xingkun Ma ${ }^{1}$, Qi Bian ${ }^{1}$
}

1 Department of Precision Instruments, Tsinghua University, Beijing 100084, China

2 College of Optical Sciences, University of Arizona, 1630 East University Boulevard, Tucson, AZ 85721, USA

hl@tsinghua.edu.cn, leihuang@email.arizona.edu, zhoucl14@mails.tsinghua.edu.cn

\begin{abstract}
:
Deformable mirror is a widely used wavefront corrector in adaptive optics system, especially in astronomical, image and laser optics. A new structure of DM--3D DM is proposed, which has removable actuators and can correct different aberrations with different actuator arrangements. A 3D DM consists of several reflection mirrors. Every mirror has a single actuator and is independent of each other. Two kinds of actuator arrangement algorithm are compared: random disturbance algorithm (RDA) and global arrangement algorithm (GAA). Correction effects of these two algorithms and comparison are analyzed through numerical simulation. The simulation results show that 3D DM with removable actuators can obviously improve the correction effects.
\end{abstract}

Key words: deformable mirror; wavefront correction; algorithm; adaptive optics

\section{Introduction}

Adaptive optics system is now widely usd in astronomical, high energy laser, medical optical instrument, and so on. Deformable mirror with piezoelectric actuators is a commonly used wavefront corrector in adaptive optics system. It is the best choice in high power laser system as its advantages such as high strock, large aperture and high power threshold. Wavefront correction ability is an important fact that we concerned. To improve wavefront correction ability of a deformable mirror, algorithm is on the one hand, and structure, especially actuator arrangement, is on the other hand. Due to the structure, actuator density can not be too high, so correction ability is limited in correction of small aperture beam and high spatial frequency aberration. To solve these questions and achieve better correction ability, a multi-reflection waveguide was proposed in Xinkun Ma's paper and better correction results were achieved through a random disturbance algorithm. ${ }^{[1,2]}$ On this basis, a kind of 3D-DM and a global arrangement algorithm is proposed in this paper and correction ability is further improved. 


\section{Three-dimensional deformable mirror}

In conventional DM, the positon of every actuator is fixed, so the arrangement mode of actuator is unchanged. Different actuator arrangement modes will influence the correction ability of DM in a certain degree. The most commonly used arrangement modes at present are square array and hexagonal array. In general, a DM with hexagonal array has better correction ability than that with square array. And for different aberration, different actuator arrangement modes have different effects on correction ability. Since actuator arrangement mode is also an important fact that will influence correction ability of DM, why not make it a variable in wavefront correction? According to this idea, a new kind of DM-3D DM is proposed in this paper. In conventional DM, every actuator has fixed position and it can only do one dimensional telescopic motion along its length. While in the new DM, in addition to the original movement, actuator can have two more dimensional movements along the mirror plane, as shown in Fig.1, so its actuator arrangement mode is changeable. As the actuators of this new DM can move in three dimensions, it is named as 3D DM.

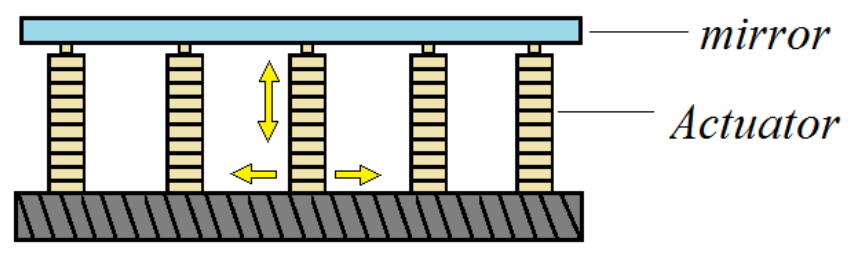

Fig.1. Schematic diagram of 3D DM

It is difficult to achieve 3D movements of every actuators in a single DM with existing technique and process. In Ma's paper ${ }^{[1,2]}$, the multi-reflection waveguide structure consists of several DM units is designed. The structure of every DM units is shown in Fig.2, the glass mirror is bonded on the base, and is driven by a single actuator. Several this units are arranged together, making up the system, and laser beam reflects on mirrors of these DM units in turn, as shown in Fig.3. As the beam incident to different areas of every mirrors, DM units can correct different part of beam, and the whole system can be equivalent to a single DM. The position of every unit is movable, and changeable actuator arrangement mode can be achieved by every units' movements. In this structure, high actuator density can be achieved in a small aperture without spatial interference of each actuator, making a better correction result in high spatial frequency aberration correction. 


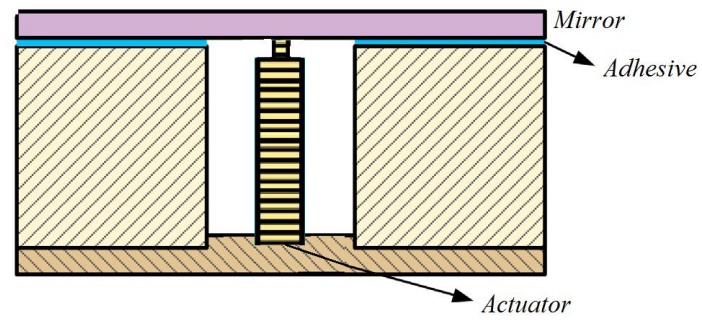

Fig.2. Structure of DM unit

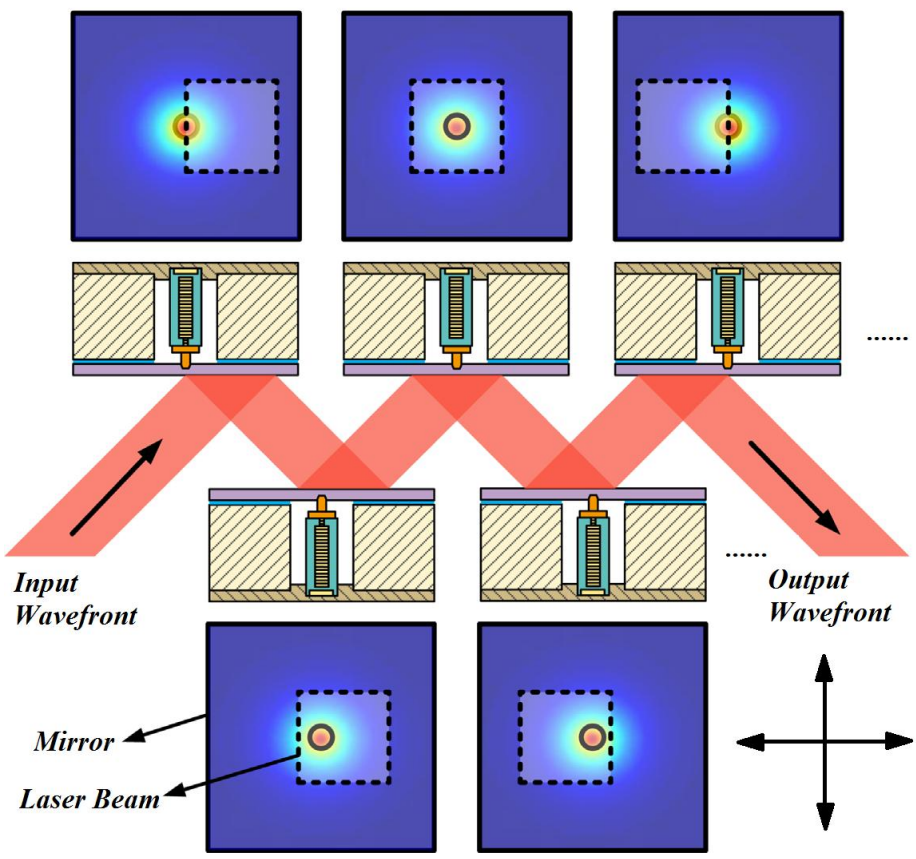

Fig.3. Waveguide structure consists of DM units

According to the multi-reflection waveguide structure, the 3D DM can be designed as discrete deformable mirrors like it. Each DM unit is driven by a single actuator. It can move in $\mathrm{x}$ and $\mathrm{y}$ two directions with a two-dimensional translation structure. Laser beam reflected by these DM units in turn and the system can be equivalent to a DM with movable actuators. A 3D DM with $n \times n$ actuators requires $n \times n$ this DM units.

\section{Control algorithm}

In aberration correction of conventional DM, control algorithm just need to calculate the control signals of every actuators. While in 3D DM, arrangement of actuators should be figured out first. Coordinate positions of every actuators should be calculated, and then control signals are calculated according to the current arrangement mode, so the control algorithm of 3D DM includes arrangement algorithm of actuators and correction algorithm. The correction algorithm is similar to that of conventional DM, sucn as least square algorithm, hill climbing algorithm and SPGD algorithm. The 
arrangement algorithm is the core of 3D DM. A proper arrangement algorithm can fully exert 3D DM's advantages and achieve better correction results.

Suppose that the working scope of $3 \mathrm{D} \mathrm{DM}$ is discretized into $n \times n$ pixels, and the wavefront to be corrected is $W$. The DM has $N$ actuators and the coordinates of them is $P=\left[\begin{array}{llll}x_{1} & x_{2} & \cdots & x_{N} \\ y_{1} & y_{2} & \cdots & y_{N}\end{array}\right]$. The influence function of $i$ th actuator is a function of $\left(x_{i}, y_{i}\right)$ : $M_{i}\left(x_{i}, y_{i}\right)=\left[m_{i 1}, m_{i 2}, \cdots m_{i n^{2}}\right]$. Influence function matrix of $N$ actuators is

$$
M(P)=\left[\begin{array}{cccc}
m_{1,1}\left(x_{1}, y_{1}\right) & m_{2,1}\left(x_{2}, y_{2}\right) & \cdots & m_{N, 1}\left(x_{N}, y_{N}\right) \\
m_{1,2}\left(x_{1}, y_{1}\right) & m_{2,2}\left(x_{2}, y_{2}\right) & \cdots & m_{N, 2}\left(x_{N}, y_{N}\right) \\
\vdots & \vdots & \ddots & \vdots \\
m_{1, n^{2}}\left(x_{1}, y_{1}\right) & m_{2, n^{2}}\left(x_{2}, y_{2}\right) & \cdots & m_{N, n^{2}}\left(x_{N}, y_{N}\right)
\end{array}\right]
$$

When 3D DM correcting aberrations, actuators redistribute. The change of actuators' coordinates to their initial positions is $\Delta P=\left[\begin{array}{llll}\Delta x_{1} & \Delta x_{2} & \cdots & \Delta x_{N} \\ \Delta y_{1} & \Delta y_{2} & \cdots & \Delta y_{N}\end{array}\right]$, and the influence function matrix now is $M(P+\Delta P)$. The task of arrangement algorithm is to find a proper $\Delta P$ to achieve better correction results.

In Ma's paper, a random disturbance algorithm(RDA) was used to calculate $\Delta P^{[1,2]}$. In this algorithm, the position of esch actuator is limited to a small area near its initial position, and can not beyond this area, so the final actuator arrangement mode is similar to the original one, every actuators just move near its initial position.

In this paper, a new algorithm--global arrangement algorithm(GAA) is used to calculate $\Delta P$. The process of GAA is as follow: for a given aberration, different actuator arrangements lead to different correction results, and it is changed continuously. In one loop, every actuators move a small distance $\mathrm{d}$ alone a random directions, and the probability of movement to every direction is all the same. The actuators' movement directions are independent of each other. That is to carry out a random movement to every actuators and a new actuator arrangement mode is achieved. If the correction result under this arrangement mode is better than that under last mode, then accept this movement and continue to the next loop; if the correction result under this arrangement mode is no better than that under last mode, then cancel this movement and continue to the next loop, so repeated. Different to RDA, the movement of esch actuator is not limited and it can be anywhere of the whole area in GAA, so the final actuator arrangement mode achieved by GAA often has a large randomness and is not related to the original arrangement mode. 
When $\Delta P$ is figured out by arrangement algorithm, the influence function matrix becomes $M(P+\Delta P)$, and then correction algorithm is used to calculate control voltages. Suppose that control voltage vector is $V=\left[v_{1}, v_{2} \cdots v_{N}\right]$, so the wavefront $W$ can be written as

$$
W=M(P+\Delta P) V=\left[\begin{array}{ccc}
m_{1,1}\left(x_{1}+\Delta x_{1}, y_{1}+\Delta y_{1}\right) & \cdots & m_{N, 1}\left(x_{N}+\Delta x_{N}, y_{N}+\Delta y_{N}\right) \\
m_{1,2}\left(x_{1}+\Delta x_{1}, y_{1}+\Delta y_{1}\right) & \cdots & m_{N, 2}\left(x_{N}+\Delta x_{N}, y_{N}+\Delta y_{N}\right) \\
\vdots & \ddots & \vdots \\
m_{1, n^{2}}\left(x_{1}+\Delta x_{1}, y_{1}+\Delta y_{1}\right) & \cdots & m_{N, n^{2}}\left(x_{N}+\Delta x_{N}, y_{N}+\Delta y_{N}\right)
\end{array}\right]\left[\begin{array}{c}
v_{1} \\
v_{2} \\
\vdots \\
v_{N}
\end{array}\right] .
$$

There is commonly no exact solution of this function, but it has a least square solution: $A^{*}=M^{+} W . M^{+}$is the generalized inverse matrix of $M$. The residual aberration after correction is $e=W-M A^{*}$. This method is called least square algorithm.

Above is the complete control algorithm of 3D DM, and the final control voltage worked out by this algorithm is

$$
\left[\begin{array}{c}
v_{1} \\
v_{2} \\
\vdots \\
v_{N}
\end{array}\right]=\left[\begin{array}{ccc}
m_{1,1}\left(x_{1}+\Delta x_{1}, y_{1}+\Delta y_{1}\right) & \cdots & m_{N, 1}\left(x_{N}+\Delta x_{N}, y_{N}+\Delta y_{N}\right) \\
m_{1,2}\left(x_{1}+\Delta x_{1}, y_{1}+\Delta y_{1}\right) & \cdots & m_{N, 2}\left(x_{N}+\Delta x_{N}, y_{N}+\Delta y_{N}\right) \\
\vdots & \ddots & \vdots \\
m_{1, n^{2}}\left(x_{1}+\Delta x_{1}, y_{1}+\Delta y_{1}\right) & \cdots & m_{N, n^{2}}\left(x_{N}+\Delta x_{N}, y_{N}+\Delta y_{N}\right)
\end{array}\right]^{+}\left[\begin{array}{c}
w_{1} \\
w_{2} \\
\vdots \\
w_{N}
\end{array}\right]
$$

\section{Numerical simulation}

In order to verify the correction ability of 3D DM, numerical simulation is carried out to compare the conventional DM and 3D DM with two arrangement algorithms. In simulation, the conventional has 49 actuators, arranged as a $7 \times 7$ square. Influence function is Gauss function. Effective aperture of beam is $100 \mathrm{~mm} \times 100 \mathrm{~mm}$. The crosslinking value is $30 \%$. In $3 \mathrm{D} \mathrm{DM}$, every $\mathrm{DM}$ units have a mirror aperture of $250 \mathrm{~mm} \times 250 \mathrm{~mm}$, and the diameter of its influence function is $100 \mathrm{~mm}$. Correction targets are 3 to 15 order Zernike aberrations. PV of 3 to 10 order aberrations are $6 \mu \mathrm{m}$, and that of 11 to 15 order aberrations are $1.5 \mu \mathrm{m}$. In simulation, these aberrations are corrected by conventional DM and 3D DM with RDA and GAA, PV and RMS of residual error after correction are compared. 

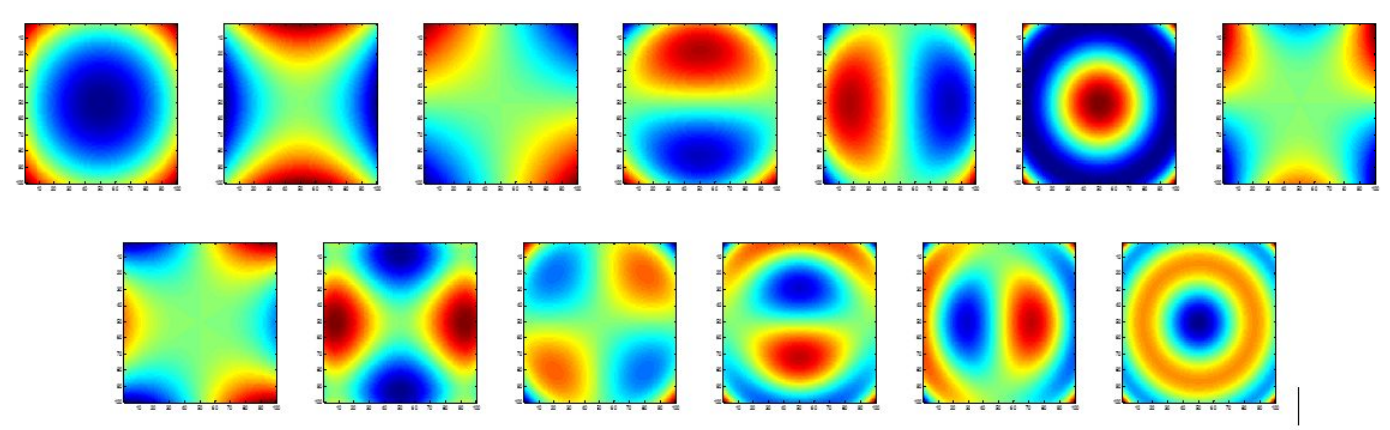

Fig.4. 3 to 15 order Zernike aberrations

\subsection{Comparison of conventional DM and 3D DM}

Table 1 and Table 2 show the PV and RMS of these three correction results, and how much the results improved by RDA and GAA than conventional DM. We can learn from the tables that compared to conventional DM, two arrangement algorithms of 3D DM can both improve the correction results to a large extent. PV and RMS of residual error can be most reduced by over $80 \%$. Those of high order aberration such as 15 order can also be reduced by over $55 \%$. And for 3 to 12 order aberrations, two algorithms of 3D DM can both reduce PV and RMS by over 70\%. So it is confirmed that the 3D DM with waveguide structure has a greater wavefront correction ability than conventional DM to a large extent.

Table 1

\begin{tabular}{|c|cccccc|}
\hline \multirow{2}{*}{$\begin{array}{c}\text { Aberration } \\
\text { order }\end{array}$} & \multicolumn{6}{|c|}{ PV } \\
\cline { 2 - 7 } & Original & Conventioal DM & RDA & Improvement & GAA & Improvement \\
\hline 3 & 6 & 0.6401 & 0.1105 & $82.74 \%$ & 0.0975 & $84.77 \%$ \\
4 & 6 & 0.6129 & 0.1600 & $73.90 \%$ & 0.1574 & $74.32 \%$ \\
5 & 6 & 0.4890 & 0.1422 & $70.92 \%$ & 0.1141 & $76.67 \%$ \\
6 & 6 & 1.3413 & 0.2553 & $80.97 \%$ & 0.2232 & $83.36 \%$ \\
7 & 6 & 1.3413 & 0.2425 & $81.92 \%$ & 0.2033 & $84.84 \%$ \\
8 & 6 & 2.3077 & 0.5349 & $76.82 \%$ & 0.4861 & $78.94 \%$ \\
9 & 6 & 0.6573 & 0.1473 & $77.59 \%$ & 0.1227 & $81.33 \%$ \\
10 & 6 & 0.6573 & 0.1240 & $81.14 \%$ & 0.1177 & $82.09 \%$ \\
11 & 1.5 & 0.2164 & 0.0560 & $74.12 \%$ & 0.0536 & $75.23 \%$ \\
12 & 1.5 & 0.4890 & 0.1467 & $70.00 \%$ & 0.1192 & $75.63 \%$ \\
13 & 1.5 & 0.8894 & 0.2983 & $66.46 \%$ & 0.2802 & $68.50 \%$ \\
14 & 1.5 & 0.8894 & 0.2934 & $67.01 \%$ & 0.2802 & $68.50 \%$ \\
15 & 1.5 & 0.8398 & 0.3767 & $55.14 \%$ & 0.3648 & $56.56 \%$ \\
\hline
\end{tabular}


Table 2

\begin{tabular}{|c|cccccc|}
\hline \multirow{2}{*}{$\begin{array}{c}\text { Aberration } \\
\text { order }\end{array}$} & \multicolumn{6}{|c|}{ RMS } \\
\cline { 2 - 7 } & Original & Conventioal DM & RDA & Improvement & GAA & Improvement \\
\hline 3 & 1.2905 & 0.0682 & 0.0126 & $81.52 \%$ & 0.0113 & $83.42 \%$ \\
4 & 1.2905 & 0.0611 & 0.0261 & $57.29 \%$ & 0.0256 & $58.10 \%$ \\
5 & 1.0203 & 0.0275 & 0.0103 & $62.60 \%$ & 0.0072 & $73.86 \%$ \\
6 & 1.4591 & 0.0890 & 0.0240 & $73.02 \%$ & 0.0215 & $75.83 \%$ \\
7 & 1.4591 & 0.0890 & 0.0215 & $75.83 \%$ & 0.0191 & $78.53 \%$ \\
8 & 1.7339 & 0.1627 & 0.0384 & $76.40 \%$ & 0.0286 & $82.43 \%$ \\
9 & 0.9050 & 0.0461 & 0.0158 & $65.75 \%$ & 0.0127 & $72.47 \%$ \\
10 & 0.9050 & 0.0461 & 0.0126 & $72.69 \%$ & 0.0123 & $73.34 \%$ \\
11 & 0.3890 & 0.0314 & 0.0067 & $78.66 \%$ & 0.0062 & $80.25 \%$ \\
12 & 0.2386 & 0.0213 & 0.0068 & $68.11 \%$ & 0.0046 & $78.43 \%$ \\
13 & 0.2905 & 0.0383 & 0.0135 & $64.77 \%$ & 0.0126 & $67.12 \%$ \\
14 & 0.2905 & 0.0383 & 0.0129 & $66.34 \%$ & 0.0125 & $67.38 \%$ \\
15 & 0.2687 & 0.0496 & 0.0204 & $58.90 \%$ & 0.0193 & $61.12 \%$ \\
\hline
\end{tabular}

\subsection{Comparison of RDA and GAA}

Fig.3 shows the fitting coefficient of 3 to 15 order aberrations corrected by conventional DM and 3D DM with RDA and GAA. Similar to the results above, 3D DM with two algotithms can effectively reduce fitting coefficient compared to conventional DM. For the two arrangement algorithm, GAA is better than RDA. Table 3 shows how much GAA reduces fitting coefficient than RDA. In general, for 3 to 9 order aberrations, GAA is much better than RDA, while for aberrations after 9 order, GAA is just a little better than RDA, improvement is under $10 \%$. In GAA, movements of actuators are not limited and more freely, that is why GAA can achieve better results than RDA. For low order aberrations, wavefront is flat and has low spatial frequency, small disterbance of actuators in RDA can not meet the position change requirements needed in wavefront correction, so GAA with more free actuator movements can improve the results to a large extent. While for high order aberrations, wavefront is complex and has high spatial frequency, small disterbance of actuators in RDA can almost meet the position change requirements needed in wavefront correction, so GAA improves just a little than RDA. In general, the correction ability of GAA is better than that of RDA. While at the condition of same correction requests, GAA needs less actuator number than RDA and saves the cost. 


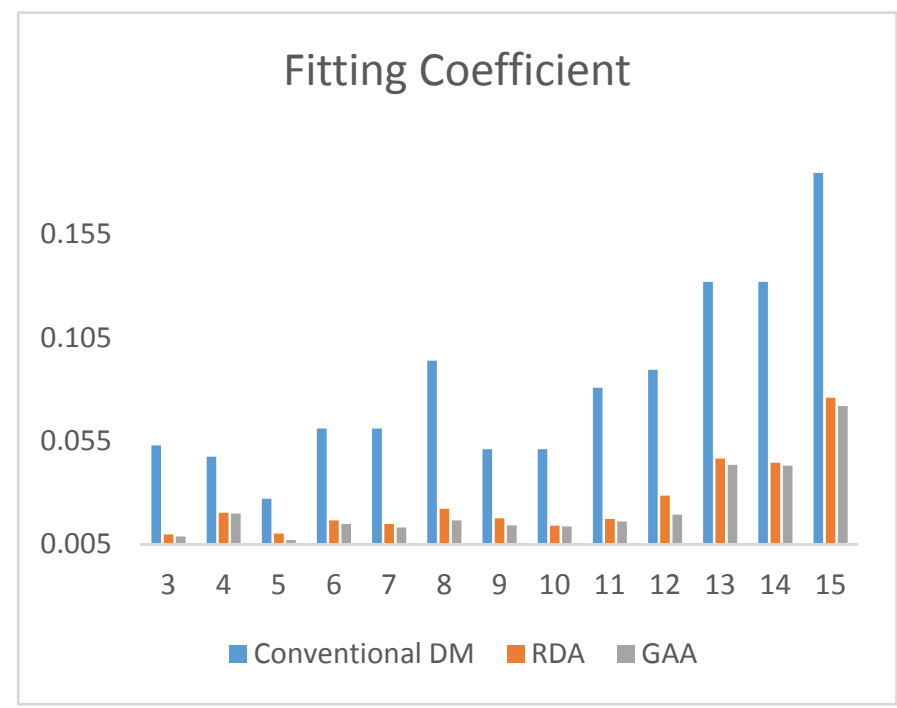

Fig.5. Fitting coefficient of three results

Table 3

\begin{tabular}{|c|c|}
\hline Aberration order & GAA impreve than RDA \\
\hline 3 & $10.3 \%$ \\
4 & $1.9 \%$ \\
5 & $30.1 \%$ \\
6 & $10.4 \%$ \\
7 & $11.2 \%$ \\
8 & $25.5 \%$ \\
9 & $19.6 \%$ \\
10 & $2.4 \%$ \\
11 & $7.5 \%$ \\
12 & $32.4 \%$ \\
13 & $6.7 \%$ \\
14 & $3.1 \%$ \\
15 & $5.4 \%$ \\
\hline
\end{tabular}

Through the above analysis and comparison of conventional DM and 3D DM with two arrangement altorithms, we can draw the conclusion that with same actuator number, correction ability:GAA $>$ RDA $>$ conventional DM; and with same correction requests, actuator number needed:GAA $<$ RDA $<$ conventional DM.

\subsection{Radius of influence function in 3D DM}

Fig.6 shows PV of residual error corrected by 3D DM with different influence function radius. We can learn from the figure that the larger radius is, the better results we can achieve. However, large control signal will be required when the radius is very 
large. In practice there is a voltage upper limit of piezoelectric actuator, so the radius can not be too large. And when the radius is over $50 \mathrm{~mm}$, the correction result is already good enough to meet the requirements. So the influence function radius should be designed reasonably according to the actual situation.

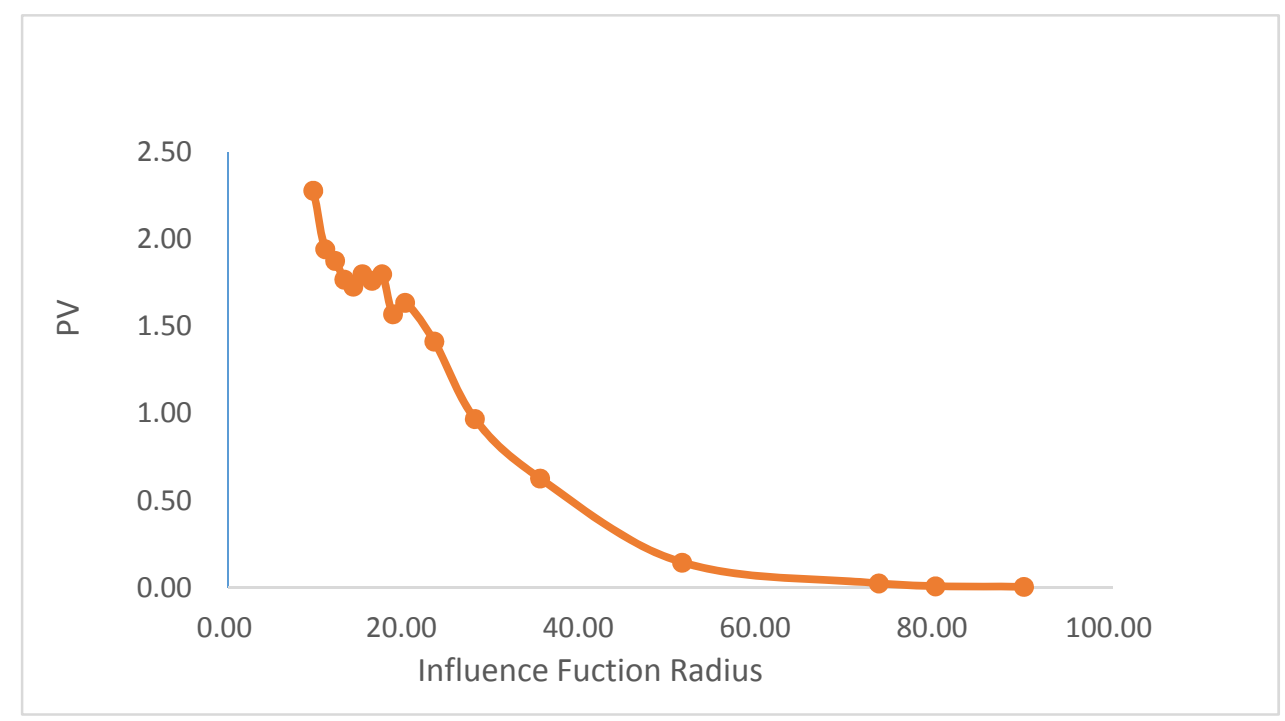

Fig.6. PV under different influence function radius

\section{Conclusion}

A novel 3D DM is described in this paper, and a kind of waveguide structure consists of several DM units is used. This system can realize 3D movement of actuators and achieve a high actuator density in a small aperture to correct high spatial frequency aberration. A new arrangement algorithm GAA is proposed and used in wavefront correction. It is also compared with conventional DM and RDA. The simulation confirmed that with same actuator number, correction ability: GAA > RDA > conventional DM; and with same correction requests, actuator number needed: GAA < RDA < conventional DM. However, there are also some problems in this 3D DM, such as beam energy loss as the light path of this system is long when actuator number is large. Relevant experiments will be carried out to further research the characteristics of 3D DM, the relationship between different aberrations and actuator arrangement modes are also to be studied.

\section{Reference}

1. X. K. Ma, L. Huang, Q. Bian, M. L. Gong, "Wavefront correction performed by a deformable mirror of arbitrary actuator pattern within a multi-reflection waveguide ," Appl. Opt. 53 (2014) 5917-5923.

2. X. K. Ma, L. Huang, Q. Bian, M. L. Gong, "Wavefront correction in a multireflection waveguide with alterable influence functions," Opt. Commun. 339 (2015) 171-176. 
3. R. G. Dekany, M. C. Britton, D. T. Gavel, B. L. Ellerbroek, G. Herriot, C. E. Max, and J.-P. Veran, "Adaptive optics requirements definition for TMT," Proc. SPIE 5490 (2004) 879-890.

4. C. A. Haynam, P. J. Wegner, J. M. Auerbach, M. W. Bowers, et al, "National Ignition Facility laser performance status," Appl. Opt. 46 (2007) 3276-3303.

5. Q. Xue, L. Huang, D. Hu, P. Yan, and M. Gong, "Research on controlling thermal deformable mirror's influence functions via manipulating thermal fields," Appl. Opt. 53 (2014) 237-243.

6. J. A. Perreault, T. G. Bifano, B. M. Levine, M. N. Horenstein, “Adaptive optic correction using microelectromechanical deformable mirrors,” Opt. Eng. 41 (2002) 561-566.

7. N. Doble, G. Yoon, L. Chen, P. Bierden, B. Singer, S. Olivier, D. R. Williams, "Use of a microelectromechanical mirror for adaptive optics in the human eye," Opt. Lett. 27 (2002) 1537-1539.

8. P. Yang, Y. Ning, X. Lei, B. Xu, X. Li, L. Dong, et al, "Enhancement of the beam quality of non-uniform output slab laser amplifier with a 39-actuator rectangular piezoelectric deformable mirror," Opt. Express 18 (2010) 7121-7130.

9. S. Piehler, B. Weichelt, A. Voss, M. A. Ahmed, T. Graf, "Power scaling of fundamental-mode thin-disk lasers using intracavity deformable mirrors," Opt. Lett. 37 (2012) 5033-5035.

10. C. A. Haynam, P. J. Wegner, J. M. Auerbach, M. W. Bowers, et al, "National Ignition Facility laser performance status," Appl. Opt. 46 (2007) 3276-3303. 